International Journal of Engineering, Science and Technology Vol. 4, No. 1, 2012, pp. 1-7

\begin{tabular}{c}
\hline \hline INTERNATIONAL \\
JOURNAL OF \\
ENGINEERING, \\
SCIENCE AND \\
TECHNOLOGY \\
\hline \hline www.ijest-ng.com \\
www.ajol.info/index.php/ijest \\
(c) 2012 MultiCraft Limited. All rights reserved
\end{tabular}

\title{
Study and analysis of wavelet based image compression techniques
}

\author{
Rajesh K. Yadav ${ }^{1}$, S.P. Gangwar ${ }^{2}$ and Harsh V. Singh ${ }^{3 *}$ \\ ${ }^{1}$ N.D. University of Agriculture \& Technology, Kumarganj, Faizabad-224 229, INDIA \\ ${ }^{2,3}$ Department of Electronics Engineering, KNIT, Sultanpur-228, INDIA \\ *Corresponding Author: e-mail: harshvikram@gmail.com, Tel +91-5362-225254, Fax. +91-5362-225254.
}

\begin{abstract}
This paper presented comprehensive study with performance analysis of very recent Wavelet transform based image compression techniques. Image compression is one of the necessities for such communication. The goals of image compression are to minimize the storage requirement and communication bandwidth. Compression is achieved by the removal of redundant data. Discrete Wavelet Transform (DWT) is a recently developed compression technique in image compression. DWT image compression includes decomposition (transform of image), Detail coefficients thresholding, and entropy encoding. This paper mainly describes the transform of an image using DWT and thresholding techniques. In this paper we have taken the standard image Lena of size 256X256 of 8 bit depth and applied DWT (haar). Then two results set are obtained by applying two different techniques of thresholding and then compare the result.
\end{abstract}

Keywords: Discrete Cosine Transform, Wavelet Transform, JPEG Compression and Entropy Encoding.

DOI: http://dx.doi.org/10.4314/ijest.v4i1.1S

\section{Introduction}

Uncompressed data such as graphics, audio and video require considerable large storage capacity and transmission bandwidth. Despite rapid growth in mass storage density and significant improvement in communication bandwidth, demand for data storage capacity and transmission bandwidth continues to outstrip the capabilities of existing technologies. One approach to mitigate this problem is to reduce the size of multimedia data transmitted over the communication channel via data compression techniques such as JPEG, JPEQ-2000 (Jena et al.). These approaches concentrate on achieving higher compression ratio without sacrificing the quality of the image. Image data compression technique, concerned with the reduction of number of bits required to store and transmit image without appreciable loss of information. A fundamental shift in the image compression approach came after the Discrete Wavelet Transform (DWT) became popular (Karam, 2008). To overcome the inefficiencies in the JPEG standard and serve emerging areas of mobile multimedia and internet communication, recently the JPEG committee has released its new image coding standard, JPEG-2000, which has been based upon DWT. The Discrete wavelet transform (DWT) has gained widespread acceptance in signal processing and image compression. Because of their inherent multi-resolution nature, wavelet-coding schemes are especially suitable for applications where scalability and tolerable degradation are important. The performance of discrete wavelet transforms based coding depends on the wavelet decomposition level and threshold value. The approximation sub-signal shows the general trend of pixel value and three detail sub-signals shows the vertical, horizontal and diagonal details or change in the image. If these details are very small then they can be set to zero without significantly changing the image. The value below which details are considered small enough to set to zero is known as the threshold. The greater the number of zeros, the greater the compression achieved. The amount of information retained by an image after compression and decompression is known as the PSNR. If the PSNR is $100 \%$ then the compression is known as lossless as the image can be reconstructed exactly (Sindhu and Rajkamal, 2009). This occurs when threshold value is set to zero, i.e. details have not been changed. If any values are changed then PSNR will be lost and is known as lossy compression. Ideally during compression the number of zeros and the PSNR will be as high as possible. However as more zeros are obtained more PSNR is lost. So a balance between the two needs to be found. 


\section{Discrete Wavelet Transform (DWT)}

The Discrete Wavelet Transform (DWT), which is based on sub-band coding. In DWT, the signal to be analyzed is passed through filters with different cutoff frequencies at different scales. Wavelets can be realized by iteration of filters with rescaling. The resolution of the signal, which is the measure of the amount of detail information in the signal, is determined by the filtering operations, and the scale is determined by up-sampling and down-sampling. The DWT is computed by successive low-pass and high-pass filtering of the discrete time-domain signal. Images are treated as two dimensional signals, they change horizontally and vertically, thus 2D wavelet analysis must be used for images (Nanavati and Panigrahi, 2005). 2D wavelet analysis uses the same 'mother wavelets' but requires an additional step at each level of decomposition. In 2D, the images are considered to be matrices with $\mathrm{N}$ rows and $\mathrm{M}$ columns. At every level of decomposition the horizontal data is filtered, and then the approximation and details produced from this are filtered on columns. At every level, four sub-images are obtained; the approximation, the vertical detail, the horizontal detail and the diagonal detail (Mahendra et al.).

\begin{tabular}{|l|r|r|}
\hline LL2 & HL2 & \\
\cline { 1 - 1 } LH2 & HH2 & HL1 \\
\hline \multicolumn{2}{|c|}{ LH1 } & HH1 \\
\hline
\end{tabular}

\section{Thresholding}

Figure 2.1: Two-level wavelet analysis

Once DWT is performed, the next task is thresholding, which is neglecting certain wavelet coefficients for level from 1 to N. There are two types of threshold: (a) Hard threshold; (b) Soft threshold

By applying hard threshold the coefficients below this threshold level are zeroed, and the output after a hard threshold is applied and defined by this equation:

$$
y_{\text {hard }}(t)=\left[\begin{array}{l}
x(t),|x(t)|>T \\
0,|x(t)| \leq T
\end{array}\right]
$$

Where $x(t)$, the input signal and $\mathrm{T}$ is the threshold. An alternative is soft threshold which is defined by the equation:

$$
y_{\text {soft }}(t)=\left[\begin{array}{l}
\operatorname{sign}(x(t))(x(t) \mid-T),|x(t)|>T \\
0,|x(t)|>T
\end{array}\right]
$$

\section{Thresholding Method}

There are two methods of thresholding: (a) Global Thresholding; (b) Level Dependent/Local Thresholding. In global thresholding, a single threshold value for all the decomposition level is used. Whereas in level dependent thresholding, different threshold values for different decomposition level are used.

\section{Entropy Encoding}

An entropy encoder further compresses the quantized values losslessly to provide better compression. It uses a model that assigns codes to symbols based on the probabilities of occurrence of the symbols so that the resultant output code stream will be smaller than the input stream. The most commonly used entropy encoders are the Huffman encoder, Run-length encoder, Arithmetic encoder, LZW encoder (Brijmohan and Mneney, 2004).

\section{Quality Measure of Image compression:}

Normally the Quality of an image compression scheme can be measured in terms of two parameters. These are:

(1) Compression efficiency: Compression efficiency is measured through compression ratio (CR). The CR can be defined as the ratio of number of zeros of the current decomposition to the number of coefficients.

(2) Distortion measurement for lossy compression: In the lossy compression algorithms, distortion measurement is used to measure the amount of information lost on the reconstruction of the original signal or image data that has been recovered from the compressed data through encoding and decoding operations. The mean square error (MSE) is one of the distortion measurements in the reconstructed data. The performance measurement parameters are signal to noise ratio (SNR) and peak signal to noise ratio (PSNR). SNR are often used for 1D data. For image data (2D) compression, the SNR is replaced by a parameter known as peak signal to noise ratio (PSNR). Mathematically Peak signal to noise ratio (PSNR) is defined as:

$$
P S N R=10 . \log _{10}\left[255^{2} / M S E \mid\right.
$$




$$
\text { Where } M S E=\frac{1}{M N}\left[\sum \sum\left(f_{i, j}-c_{i, j}\right)^{2}\right]
$$

Generally when PSNR is $40 \mathrm{~dB}$ or greater, then the original and the reconstructed images are virtually indistinguishable by human observers.

\section{The Proposed Algorithm}

The introduced algorithm is simulated in matlab7.1 and described as follows:
(1) $X=$ input image of size of $256 \times 256 \times 8$
(2) $\mathrm{N}=$ Enter the decomposition level
(3) $\mathrm{W}=$ Enter the name of wavelet
(4) Find the DWT coefficients [CA, CH, CV, CD] of image X
(5) $\mathrm{T}=$ Enter the global/local threshold value
(6) Decompose the approximate coefficient [CA] to N level by W wavelet.
(7) Find the compression ratio.
(8) Reconstructs the matrix $X$ based on the multi-level wavelet
(9) Compute Mean Square Error
(10) Compute Peak Signal to Noise Ratio

\section{Experimental Result}

\subsection{Result Set-1 (Global Thresholding)}

We have implemented the proposed algorithm, using 'haar' DWT and applying global threshold on standard Lena image of size 256x256 of 8 bit depth at six decomposition levels (1, 2, 3, 4, 5, and 6). Each decomposition level is observed with different threshold value $\boldsymbol{T}$. Final Results are observed in terms of CR and PSNR.

Table 8.1: Discrete Wavelet Transform (Global Thresholding)

\begin{tabular}{|c|c|c|c|}
\hline DL (N) & Threshold value (T) & CR (in \%) & PSNR (in dB) \\
\hline 1 & 5 & 34.8938 & 43.1323 \\
\hline 1 & 8 & 44.5557 & 39.9808 \\
\hline 1 & 10 & 48.8037 & 38.5458 \\
\hline 1 & 12 & $51.9897^{`}$ & 37.4188 \\
\hline 1 & 15 & 55.3345 & 36.0740 \\
\hline 2 & 5 & 39.3005 & 41.9366 \\
\hline 2 & 8 & 50.8667 & 38.6721 \\
\hline 2 & 10 & 56.0730 & 37.1925 \\
\hline 2 & 12 & 60.0281 & 36.0013 \\
\hline 2 & 15 & 64.5704 & 34.5704 \\
\hline 3 & 5 & 39.7644 & 41.6837 \\
\hline 3 & 8 & 51.5686 & 38.3637 \\
\hline 3 & 10 & 56.8909 & 36.8467 \\
\hline 3 & 12 & 60.9375 & 35.6293 \\
\hline 3 & 15 & 65.3870 & 34.1674 \\
\hline 4 & 5 & 39.7766 & 41.5872 \\
\hline 4 & 8 & 51.5930 & 38.2758 \\
\hline 4 & 10 & 56.9153 & 36.7422 \\
\hline 4 & 12 & 60.9680 & 35.0529 \\
\hline 4 & 15 & 65.4480 & 34.0529 \\
\hline 5 & 5 & 39.7766 & 41.5703 \\
\hline 5 & 8 & 51.5930 & 38.2482 \\
\hline 5 & 10 & 56.9153 & 36.7299 \\
\hline 5 & 12 & 60.9680 & 35.4971 \\
\hline 5 & 15 & 65.4480 & 34.0284 \\
\hline 6 & 5 & 39.7766 & 41.5701 \\
\hline 6 & 8 & 51.5930 & 38.2417 \\
\hline 6 & 10 & 56.9153 & 36.7227 \\
\hline 6 & 12 & 60.9680 & 35.4921 \\
\hline 6 & 15 & 65.4480 & 34.0312 \\
\hline
\end{tabular}




\subsection{Result Set-2 (Level Dependent Thresholding)}

Again we implemented the proposed algorithm, using 'haar' DWT and applying level dependent threshold on the same image. Each decomposition level is observed with different threshold value $\boldsymbol{T}$.

Table 8.2: Discrete Wavelet Transform (Level Dependent Thresholding)

\begin{tabular}{|c|c|c|c|c|c|c|c|c|}
\hline$D L(N)$ & \multicolumn{6}{|c|}{ Threshold value (T) } & $C R$ & $P S N R($ in $d b)$ \\
\hline \multicolumn{9}{|c|}{ Threshold Value range from 1 to 16} \\
\hline \multirow{3}{*}{1} & \multicolumn{6}{|c|}{1} & & \\
\hline & \multirow{2}{*}{\multicolumn{6}{|c|}{$\begin{array}{l}2 \\
3\end{array}$}} & 16.6931 & 58.6808 \\
\hline & & & & & & & & \\
\hline \multirow{3}{*}{2} & \multicolumn{2}{|r|}{1} & \multicolumn{4}{|c|}{2} & \multirow{3}{*}{19.4031} & \multirow{3}{*}{57.6245} \\
\hline & \multirow{2}{*}{\multicolumn{2}{|c|}{$\frac{2}{3}$}} & \multicolumn{4}{|c|}{3} & & \\
\hline & & & \multicolumn{4}{|c|}{4} & & \\
\hline \multirow{3}{*}{3} & \multirow{2}{*}{$\frac{1}{3}$} & \multirow{2}{*}{\multicolumn{2}{|c|}{$\frac{2}{4}$}} & & \multicolumn{2}{|c|}{3} & \multirow{3}{*}{26.1353} & \multirow{3}{*}{52.8423} \\
\hline & & & & & 5 & & & \\
\hline & 5 & & 6 & & 7 & & & \\
\hline & 1 & & 2 & 3 & 4 & & & \\
\hline 4 & 4 & & 5 & 6 & 7 & & 31.3660 & 49.7886 \\
\hline & 7 & & 8 & 9 & 10 & & & \\
\hline & 1 & 2 & 3 & & 4 & 5 & & \\
\hline 5 & 5 & 6 & 7 & & 8 & 9 & 35.3394 & 47.5829 \\
\hline & 9 & 10 & 1 & & 1 & 1 & & \\
\hline & & & 1 & 2 & 2 & 3 & & \\
\hline & 1 & 2 & 3 & 4 & 5 & 6 & & \\
\hline 6 & 6 & 7 & 8 & 9 & 1 & 1 & 38.3667 & 45.9178 \\
\hline & & & & & 0 & 1 & & \\
\hline & 1 & 1 & 1 & 1 & 1 & 1 & & \\
\hline & 1 & 2 & 3 & & 5 & 6 & & \\
\hline & & & reshold & d Val & ue ran & nge $\mathrm{fr}$ & rom 5 to 15 & \\
\hline & & & 5 & & & & & \\
\hline 1 & & & 6 & & & & 38.4827 & 48.4153 \\
\hline & & & 7 & & & & & \\
\hline & & 5 & & & 6 & & & \\
\hline 2 & & 6 & & & 7 & & 44.0308 & 47.5757 \\
\hline & & 7 & & & 8 & & & \\
\hline & 5 & & 6 & & 7 & & & \\
\hline 3 & 7 & & 8 & & 9 & & 48.0896 & 45.9094 \\
\hline & 9 & & 10 & & 11 & & & \\
\hline & 5 & & 6 & 7 & & 8 & & \\
\hline 4 & 8 & 9 & 9 & 1 & 1 & 1 & 50.6042 & 44.6432 \\
\hline & & & $\underline{0}$ & 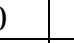 & & & & \\
\hline & 11 & 1 & 1 & 1 & & 4 & & \\
\hline & & 2 & 3 & & & & & \\
\hline & 5 & 6 & 7 & 8 & & 9 & & \\
\hline 5 & 9 & 1 & 1 & 1 & & 13 & 52.6794 & 43.5577 \\
\hline & & 0 & 1 & 2 & & & & \\
\hline & 1 & 1 & 1 & 1 & & 17 & & \\
\hline & 3 & 4 & 5 & 6 & & & & \\
\hline & 5 & 6 & 7 & 8 & 9 & 1 & & \\
\hline 6 & & & & & & 0 & 54.2908 & 42.7153 \\
\hline & 1 & 1 & 1 & 1 & 1 & 1 & & \\
\hline & 0 & 1 & 2 & 3 & 4 & 5 & & \\
\hline & 1 & 1 & 1 & 1 & 1 & 2 & & \\
\hline & 5 & 6 & 7 & 8 & 9 & 0 & & \\
\hline & & Thre & reshold & Valu & ue rang & ge fro & om 10 to & \\
\hline
\end{tabular}




\begin{tabular}{|c|c|c|c|c|c|c|c|c|}
\hline 1 & \multicolumn{6}{|c|}{$\begin{array}{l}11 \\
12\end{array}$} & 50.4639 & 43.3501 \\
\hline \multirow{3}{*}{2} & \multicolumn{2}{|r|}{10} & \multicolumn{4}{|c|}{11} & \multirow{3}{*}{58.4534} & \multirow{3}{*}{42.4325} \\
\hline & \multirow{2}{*}{\multicolumn{2}{|c|}{$\frac{11}{12}$}} & \multicolumn{4}{|c|}{12} & & \\
\hline & & & \multicolumn{4}{|c|}{13} & & \\
\hline \multirow{3}{*}{3} & \multicolumn{2}{|c|}{$\frac{12}{10}$} & \multirow{2}{*}{$\frac{11}{13}$} & & \multicolumn{2}{|c|}{12} & \multirow{3}{*}{61.0962} & \multirow{3}{*}{41.5798} \\
\hline & \multicolumn{2}{|c|}{12} & & & \multicolumn{2}{|c|}{14} & & \\
\hline & \multicolumn{2}{|c|}{14} & \multicolumn{2}{|c|}{15} & \multicolumn{2}{|c|}{16} & & \\
\hline \multirow{6}{*}{4} & 10 & & 1 & 1 & & 13 & \multirow{6}{*}{62.3779} & \multirow{6}{*}{40.9945} \\
\hline & & & & 2 & & & & \\
\hline & 13 & & 1 & 1 & & 16 & & \\
\hline & & & & 5 & & & & \\
\hline & \multirow[t]{2}{*}{16} & & 1 & 1 & \multirow{2}{*}{\multicolumn{2}{|c|}{19}} & & \\
\hline & & & & 8 & & & & \\
\hline \multirow{6}{*}{5} & 1 & 1 & 1 & 1 & & 14 & \multirow{6}{*}{63.5986} & \multirow{6}{*}{40.4000} \\
\hline & 0 & 1 & 2 & 3 & & & & \\
\hline & 1 & 1 & 1 & 1 & & 18 & & \\
\hline & 4 & 5 & 6 & 7 & & & & \\
\hline & 1 & 1 & 2 & 2 & & 22 & & \\
\hline & 8 & 9 & 0 & 1 & & & & \\
\hline \multirow{6}{*}{6} & 1 & 1 & 1 & 1 & 1 & 1 & \multirow{6}{*}{64.7461} & \multirow{6}{*}{39.7890} \\
\hline & 0 & 1 & 2 & 3 & 4 & 5 & & \\
\hline & 1 & 1 & 1 & 1 & 1 & 2 & & \\
\hline & 5 & 6 & 7 & 8 & 9 & 0 & & \\
\hline & 2 & 2 & 2 & 2 & 2 & 2 & & \\
\hline & 0 & 1 & 2 & 3 & 4 & 5 & & \\
\hline
\end{tabular}

\section{Graphical Representation}

9.1 Result Set-1

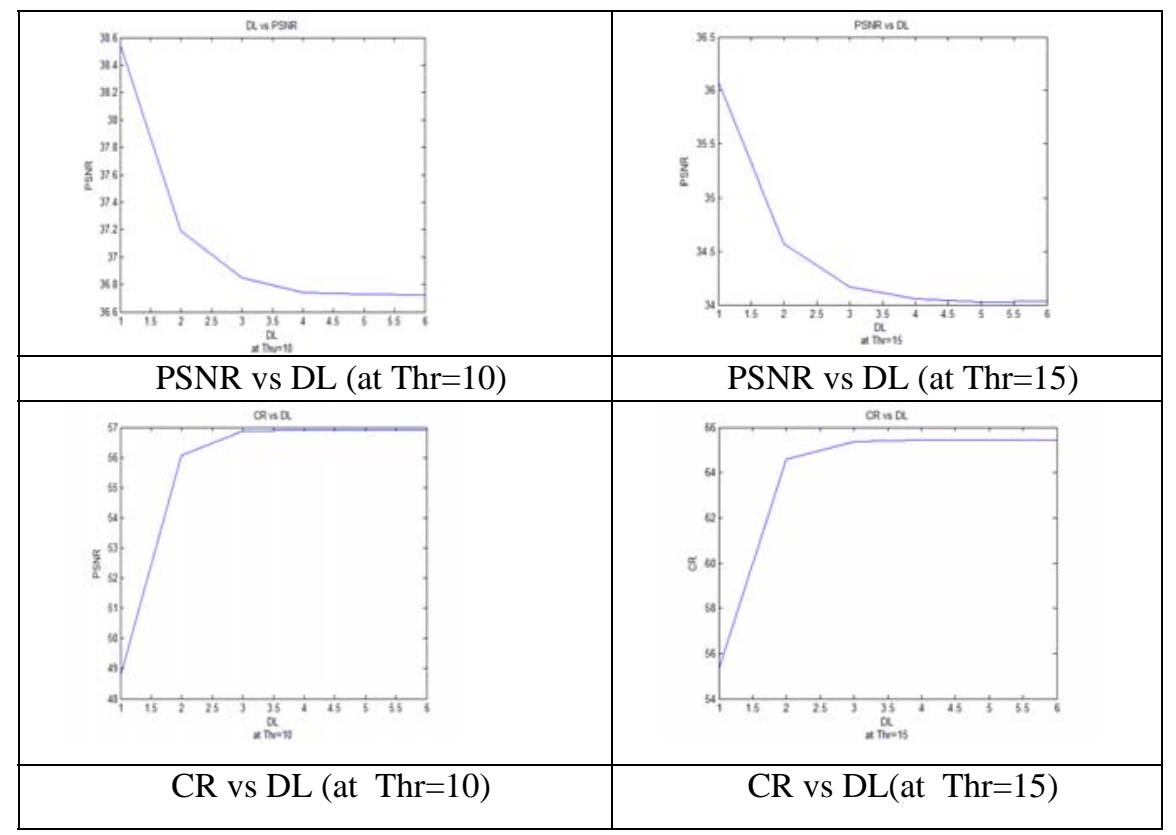




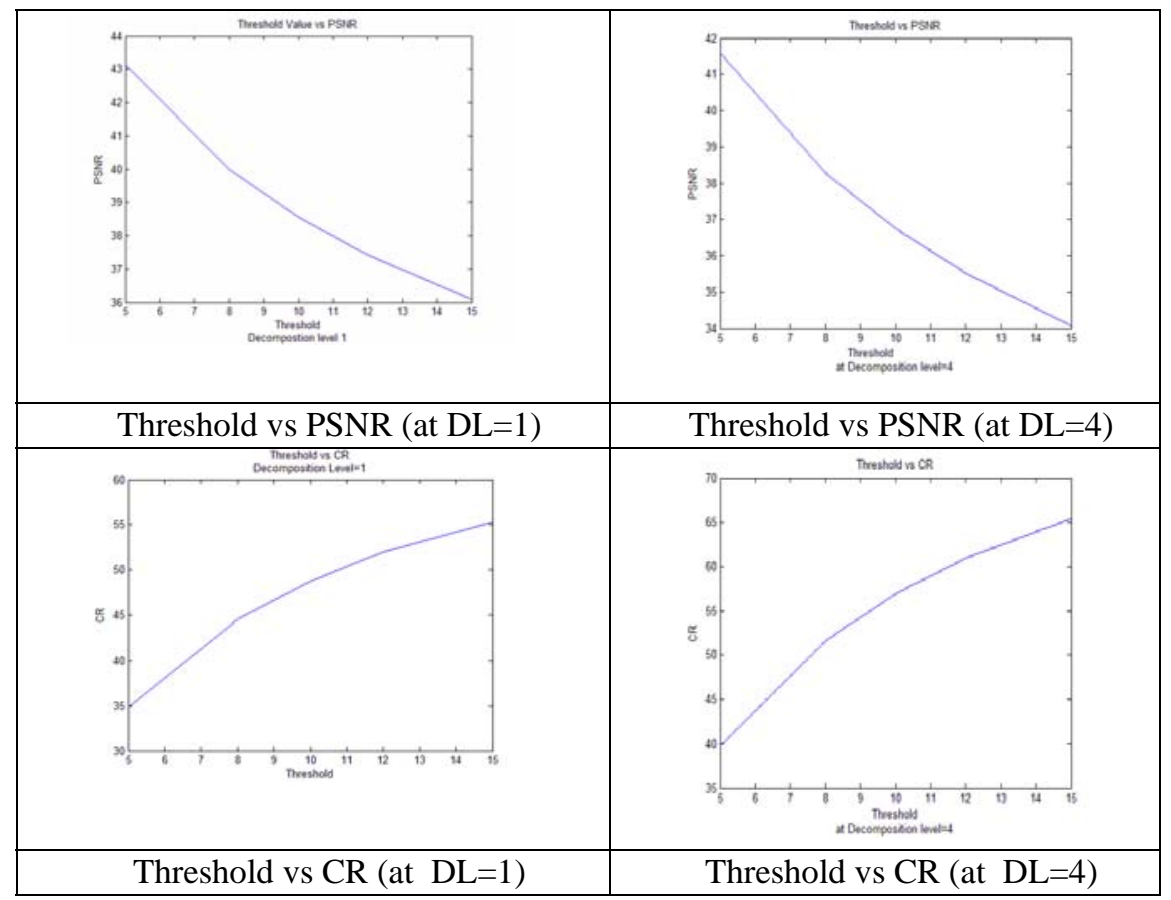

9.2 Result Set-2

Figure 9.1: Graphical representation of result using global threshold

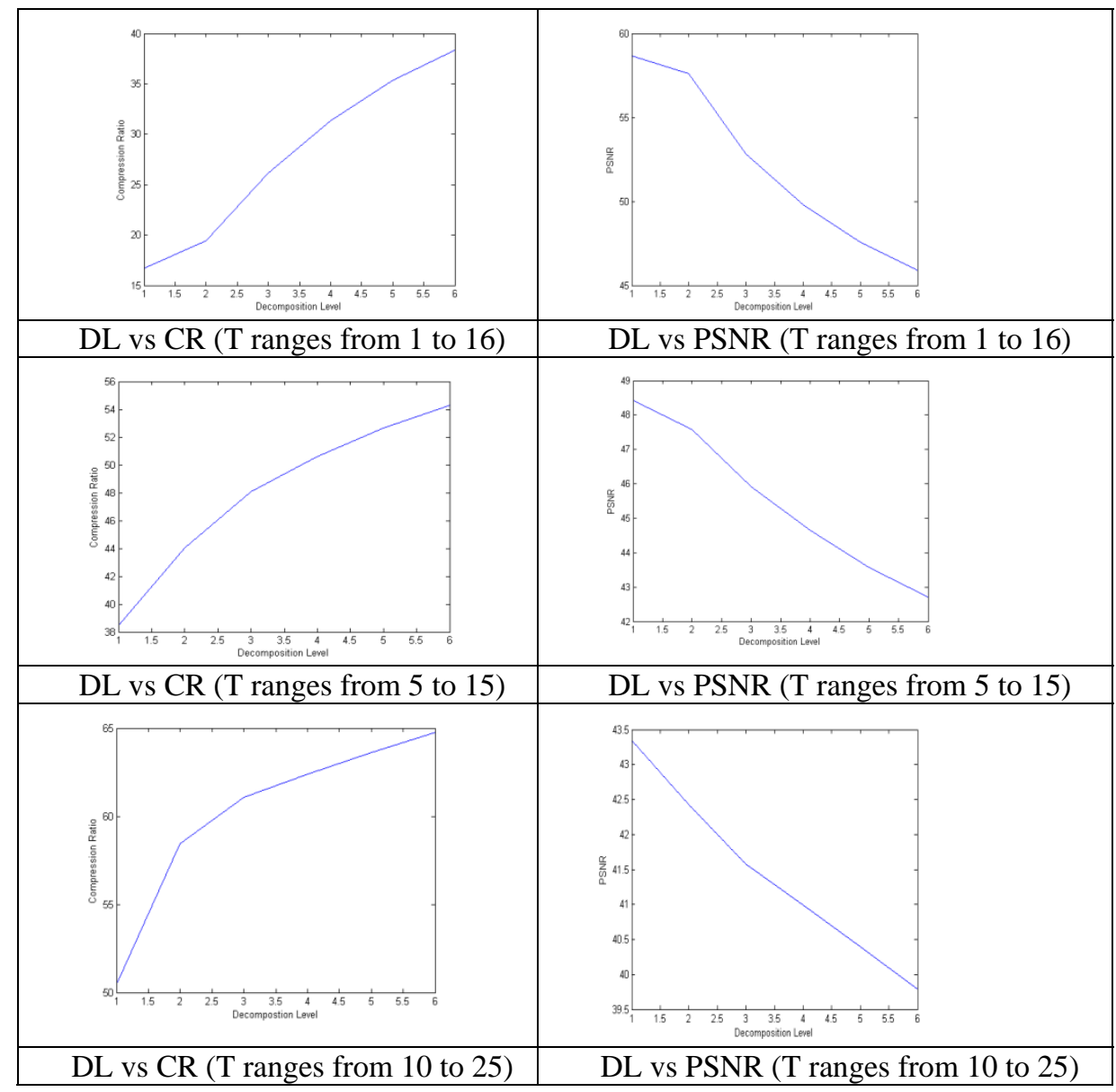

Figure 9.2: Graphical representation of result using level dependent threshold 


\section{Conclusion}

The higher the decomposition level the higher percentage of zeros obtained with no thresholding. This is because decomposing to greater levels means that a higher percentage of coefficients come from detail sub-signals. Detail sub-signals generally have a smaller range of values than the approximation sub-signals, ideally zero values. Therefore this pattern shows that as decomposition level increases, more detail is filtered out with value zero. Although global thresholding can be used successfully to compress images it is difficult to find a global threshold that will give near optimal results because of how the different detail sub-signals differ. Global thresholding leads to unnecessary loss in PSNR. Therefore it is more logical to use local thresholds. The thresholding strategy was improved by using a local rather than global technique the results only used a very small proportion of the possible threshold combinations. In global thresholding the best trade-off between PSNR and compression is provided by decomposing to levels 3 to 4 and thresholded to a value range from 5 to 7 . On the other hand in level dependent thresholding the best trade-off between PSNR and compression is provided by decomposing to levels 3 to 5 and thresholded to a value range from 5 to 20 .

\section{References}

Brijmohan Y. and Mneney S.H., 2004. "Benchmark for modern wavelet based still image compression" (http://www.ias.ac.in/resonance/Dec2004/pdf/Dec2004p08-13.pdf)

Jena G., Singh R.B.. "Image Compression using Wavelet Transform, DCT and Performance Comparison"( http://drgjena.co.cc/2Image\%20Compression.pdf)

Karam J., 2008. “A new approach in wavelet based speech compression”.(http://www.wseas.us/elibrary/conferences/2008/corfu/mnw/mnw35.pdf), pg 228-233,ISSN:1790-2769

Mahendra V.P., Arjun M.R., Yadav H.S., Manoj M.S. "Implementation of advance Image Compression Algo. using WT" (http://sidharth0384.googlepages.com/Report.pdf)

Nanavati S. P. and Panigrahi P.K., 2005. "Wavelet: Applications to Image Compression-II”. (http://www.ias.ac.in/ resonance/Mar2005/pdf/Mar2005p19-27.pdf)

Sindhu M., Rajkamal R., 2009. "Image and its compression techniques” International Journal of Recent Trends in Engineering, Vol 2, No. 4, pp. 71-75.

\section{Biographical notes}

Rajesh K. Yadav received B.Tech. (Computer Sci. \& Engg.) from Dr. Ram Manohar Lohia Avadh University, Faizabad, Uttar Pradesh, India in 2004 and M.Tech.(Electronics Engg.) from Kamla Nehru Institute of Technology, Sultanpur, India in 2011. Presently he is presently working as a programmer in N.D. University of Agriculture \& Technology, Kumarganj, Faizabad, Uttar Pradesh, India.

S.P.Gangwar received B.E. (Electronics \& Communication Engineering) from M.J.P. Rohilkhand, Bareilly, India and M.Tech from G.B.P.U.Ag. \& T,Pantnagar, India. Presently he is working as Assistant Professor in autonomous government engineering college, KNIT Sultanpur. His areas of teaching and research interest are information security, microwave and antennas. He has chaired technical sessions at national conferences / seminars.

Dr. Harsh Vikram Singh received B.Tech. (Hon.) degree in Electronics Engineering from IET- PU, India and Ph. D. from IT-BHU, Varanasi, India. Presently he is working as Assistant Professor in autonomous government engineering college, KNIT Sultanpur. His areas of teaching and research interest are information security, digital system design, image processing and embedded system. He has chaired technical sessions at international and national conferences / seminars. He has authored a book on "Information Hiding Techniques" for Lambert Academic Publication, Germany and 36 research papers published in reputed international and national journals, conference / seminar proceedings and supervised a good number of M. Tech. dissertations and Ph. D. theses. Dr. Singh has reviewed research papers for IEEE transactions and other reputed international journals. His papers have received best paper awards in international conferences. He is Member of WASET Scientific and Technical Committee on Natural and Applied Sciences (UK), International Association of Engineers (IAENG-USA), indianscience (India) and Universal Association of Computer and Electronics Engineers, India.

Received January 2012

Accepted February 2012

Final acceptance in revised form March 2012 